\title{
Identification and Functional Characterization of a Novel Bipartite Nuclear Localization Sequence in ARID1A
}

\author{
Nicholas W. Bateman ${ }^{\mathrm{a}, \mathrm{b}}$, Yutaka Shojic, Kelly A. Conrads ${ }^{\mathrm{a}}$, Kevin D. Stroop ${ }^{\mathrm{a}}$, Chad A. \\ Hamilton $^{\mathrm{a}, \mathrm{b}, \mathrm{d}, \mathrm{e}}$, Kathleen M. Darcy ${ }^{\mathrm{a}, \mathrm{b}}$, G. Larry Maxwell ${ }^{\mathrm{f}}$, \\ John I. Risinger ${ }^{\mathrm{c}}$ and Thomas P. Conrads $\mathrm{s}^{\mathrm{a}, \mathrm{b}, \mathrm{d}^{*}}$
}

${ }^{a}$ Women's Health Integrated Research Center at Inova Health System, Gynecologic Cancer Center of Excellence, Annandale, VA, 22003, USA

${ }^{\text {b}}$ The John P. Murtha Cancer Center, Walter Reed National Military Medical Center, 8901 Wisconsin Avenue, Bethesda, MD 20889, USA

'Department of Obstetrics, Gynecology and Reproductive Biology, Michigan State University, Grand Rapids, MI, 49503, USA

${ }^{\mathrm{d} G y n e c o l o g i c}$ Oncology Service, Department of Obstetrics and Gynecology, Walter Reed National Military Medical Center, 8901 Wisconsin Ave, Bethesda, MD, 20889, USA

${ }^{\mathrm{e}}$ Department of Obstetrics and Gynecology, Uniformed Services University of the Health Sciences, Bethesda, MD 20814, USA

fDepartment of Obstetrics and Gynecology, Inova Fairfax Hospital, Falls Church, VA, 22042, USA

Corresponding Author: Thomas P. Conrads, PhD, 3289 Woodburn Rd, Suite 375, Annandale, VA, 22003. Ph: 703-207-3357; Fax: 703-560-0919; Email: conrads@ whirc.org 


\begin{abstract}
AT-rich interactive domain-containing protein 1A (ARID1A) is a recently identified nuclear tumor suppressor frequently altered in solid tumor malignancies. We have identified a bipartitelike nuclear localization sequence (NLS) that contributes to nuclear import of ARID1A not previously described. We functionally confirm activity using GFP constructs fused with wildtype or mutant NLS sequences. We further show that cyto-nuclear localized, bipartite NLS mutant ARID1A exhibits greater stability than nuclear-localized, wild-type ARID1A. Identification of this undescribed functional NLS within ARID1A contributes vital insights to rationalize the impact of ARID1A missense mutations observed in patient tumors.
\end{abstract}

\title{
Keywords
}

ARID1A, nuclear localization sequence, tumor suppressor, trafficking 


\section{Introduction}

The AT-rich interactive domain-containing protein 1A (ARIDIA/ BAF250a) gene product is a $\sim 242 \mathrm{kDa}$ protein and belongs to the SWItch/Sucrose Non-Fermentable (SWI/SNF) chromatin remodeling family. ${ }^{1}$ ARID1A harbors a DNA-binding ARID domain ${ }^{2}$ and additional functional domains associated with transcriptional regulation, such as a hypermethylated in cancer 1 (HIC1) binding domain ${ }^{3}$ and multiple leucine-rich steroid receptor binding (LXXLL) motifs (Supplemental Figure 1). ARID1A loss of function mutations have been reported in multiple tumor types including breast, colon, lung, renal, pancreatic, bladder, endometrial, cervical and ovarian adenocarcinomas, as well as endocervical-type atypical proliferative tumors of the ovary. ${ }^{4-6}$ To date, 654 unique mutations have been reported for ARID1A across 28 primary cancer subtypes derived from various public data sets. ${ }^{7}$ Although many ( 77\%) of identified mutations result in frame-shifts, in-frame insertions, deletions, or splice alterations producing an unstable protein product and loss of function, approximately $23 \%$ are characterized as missense mutations, the functional manifestation of which are poorly understood. Thus, it is vital to expand the knowledgebase of functionally-active domains within ARID1A to better understand the potential ramifications of a given missense mutation observed in tumor specimens. With this goal in mind, we set out to expand upon recent efforts detailing functional domains responsible for nuclear trafficking of ARID1A. ${ }^{8}$ Here we have identified a bipartite-like nuclear localization sequence (NLS) that contributes to nuclear import of ARID1A not previously described. 


\section{Materials and Methods}

\section{Cell Culture and Reagents}

HEC1A and TOV21G cells were obtained commercially (ATCC, Manassas, VA). Cell lines were maintained in complete DMEM-F12 media (ATCC) and supplemented with 10\% tetracycline-free FBS and 1\% penicillin/streptomycin (Pen/Strep). Doxycycline was obtained from Clontech and MG-132 from Sigma-Aldrich.

Generation of a tetracycline-inducible, human ARIDIA expression construct and ARIDIA nuclear localization sequence (NLS) mutational variants.

A construct encoding the full-length, human ARID1A open-reading frame (ORF) was obtained commercially (Origene cat\# RC218151). The 5'and 3'-ends of the ARID1A ORF were modified by the addition of linker sequences including a 5'-AgeI restriction site and 3'-AscI site. The ARID1A ORF was then sub-cloned via AgeI and MluI sites into a tetracycline-inducible lentiviral DNA construct, pTRIPZ (Dharmacon/ GE Healthcare), immediately downstream of the tetracycline response element (TRE). The ARID1A monopartite-like NLS mutant variant was generated by replacing a region of the ARID1A ORF spanning BsrGI (T4163-A4168) and a BstBI (T5963-A5968) restriction endonuclease sites with a commercially synthesized (GeneArt, Invitrogen) variant sequence in which R1656-R1658 were converted to T1656-T1658 by introducing the following mutations, G4967C, G4970C, C4972A and G4973C. The ARID1A bipartite-like NLS mutant variant was generated by replacing a region of the ARID1A ORF spanning a EcoRI (G3606-C3611) and BsrGI (T4163-A4168) restriction endonuclease site with a commercially synthesized (GeneArt, Invitrogen) variant sequence in which K1370, R1371 and 
R1383 were converted to T1370, T1371 and T1383 by introducing the following mutations A4109C, C4111A, G4112C, C4147A, G4148C. All constructs were confirmed by DNA sequencing.

Generation of wildtype and mutated GFP-NLS fusion constructs:

Green fluorescent protein (GFP) \& ARID1A nuclear localization sequence (NLS) candidate fusion constructs were constructed for the Gateway cloning system using the Vector NTI software suite (Invitrogen). Specifically, Gateway entry vectors containing Glycine (G)/ Serine (S) x 10 linker sequences followed by wildtype or mutated ARID1A NLS candidate sequences were designed using the GeneArt assembly tool in Vector NTI and were then commercially synthesized and subcloned into pDONR221 entry vectors (GeneArt, Invitrogen). Entry vectors were then LR recombined into the expression vector, pcDNA-DEST53, resulting in a Cycle 3 GFP ORF, c-terminally tagged with a GS-linker and the candidate ARID1A NLS sequence of interest. All constructs were confirmed by DNA sequencing.

Immunofluorescence analyses of cell lines transiently transfected with ARIDIA or GFP-NLS fusion constructs.

For ARID1A analyses, cells were plated in 8-well chamber slides (Corning ${ }^{\circledR}$ BioCoat ${ }^{\mathrm{TM}}$ Poly-DLysine 8 Well CultureSlide) and sub-confluent cells were transfected with $1.0 \mu \mathrm{g}$ of ARID1AWT or ARID1A-Bipartite NLS on day two followed by incubation for $24-48$ hours at $37^{\circ} \mathrm{C}$. Cell media was then changed, supplemented with $1.0 \mu \mathrm{g} / \mathrm{mL}$ of doxycycline and cultures were incubated an additional three days. Chamber slides were then washed with 1X PBS, fixed in 4\% formaldehyde/ 1X PBS for 15 minutes at room temperature (RT), washed with 1X PBS, 
permeabilized with $0.5 \%$ Triton X-100, washed again with $1 \mathrm{X}$ PBS, blocked with $2.0 \%$ bovine serum albumin in 1X PBS for $20 \mathrm{~min}$ at RT and incubated with anti-ARID1A, rabbit polyclonal antibody overnight (Sigma-Aldrich, HPA005456, 1:1000). Slides were then washed three times with $1 \mathrm{X}$ PBS for $10 \mathrm{~min}$ at RT, incubated with FITC-conjugated, goat anti-rabbit secondary antibody (1:1000, Cell Signalling) in $2.0 \%$ BSA/ 1X PBS for three hours, washed thrice as above with the final wash being supplemented with rhodamine phalloidin stain (Thermo Fisher) and Hoechst 3342 stain (NucBlue, Thermo Fisher) as per manufacturer's recommendations. Fluorescence images were obtained at 40X magnification on an Olympus IX51 inverted fluorescence microscope using cellSens Dimension Software ver. 1.6 (Olympus).

For GFP-NLS fusion analyses, cells were plated in 8-well chamber slides on day one and subconfluent cells were transfected with $1.0 \mu \mathrm{g}$ of GFP-NLS fusion construct variants followed by incubation for $72-96$ hours at $37^{\circ} \mathrm{C}$. Slides were then washed twice with $1 \mathrm{X}$ PBS, with the final wash being supplemented with rhodamine phalloidin stain (Thermo Fisher) and Hoechst 3342 stain (NucBlue, Thermo Fisher) as per manufacturer's recommendations. Fluorescence images were obtained as described above.

\section{ARIDIA protein stability studies}

Sub-confluent cells were transfected with $1.0 \mu \mathrm{g}$ of ARID1A-WT or ARID1A-Bipartite NLS mutant DNA vectors using Trans-IT LT1 transfection reagent as per manufacturer's recommendations (Mirus). Cell media was changed and supplemented with $1.0 \mu \mathrm{g} / \mathrm{mL}$ of doxycycline on day two and cultures were incubated an additional three days. Cultures were then treated with $10 \mu \mathrm{M}$ MG132 for $3 \mathrm{~h}$ followed by cell lysis (1\% SDS, 10mM Tris-HCL pH 7.4). 
Equivalent amounts of cell lysates were resolved on 4-15\% mini-PROTEIN TGX gels and transferred to PVDF membranes. Membranes were blocked for $1 \mathrm{~h}$ with 5\% non-fat dry milk in 1X TBST and incubated with primary antibody overnight at $4{ }^{\circ} \mathrm{C}$. Secondary antibody was incubated for $3 \mathrm{~h}$ at ambient temperature followed by incubation in SuperSignal West Dura Chemiluminescent Substrate (ThermoFisher Scientific) for $5 \mathrm{~min}$. Antibodies and concentrations were used as follows: anti-ARID1A rabbit polyclonal (Sigma-Aldrich, HPA005456, 1:1000), anti-beta actin rabbit polyclonal (ab9485, Abcam, 1:1,000), HRP-linked goat anti-rabbit IgG (Cell Signaling Technologies. 1:1000). Images were acquired using a ChemiDoc XRS+ system (Bio-Rad). 


\section{Results}

A nuclear export sequence for ARID1A spanning amino acid residues 2004-2021 was recently described in work that further identified cytoplasmic and nuclear populations of ARID1A that exhibit distinct protein stability dynamics. ${ }^{8}$ It was noted that ARID1A contains a potential nuclear localization sequence (NLS) reminiscent of a class II monopartite NLS sequence [e.g. $\mathrm{K}(\mathrm{K} / \mathrm{R}) \mathrm{X}(\mathrm{K} / \mathrm{R})]^{9}$ spanning amino acid residues 1651-1657 (PVLKQRR), though no confirmatory evidence was provided to support functional activity of this motif (Fig. 1). We generated full length ARID1A expression constructs harboring either the wild-type NLS or a mutated NLS harboring threonyl residues in place of the arginyl residues (e.g. PVLKQTTT) (see Supplemental Methods) and assessed subcellular localization patterns in ARID1A mutant cell line models, including a model of clear-cell ovarian carcinoma (TOV21G, frame-shift mutations in ARIDIA, c.1650dupc/ c.2272delC) ${ }^{10}$ and a model of endometrial cancer (HEC1A, nonsense mutations in ARID1A, 5876C $>$ CT/ $6716 \mathrm{C}>\mathrm{CT})^{11}$. Somewhat unexpectedly, assessment of ARID1A by immunofluorescence showed that mutation of this region had no impact on the subcellular localization of the expression construct (Fig. 2A-B, D-E). These results were further validated using an expression conjugate consisting of green fluorescent protein (GFP) conjugated Cterminally to the putative monopartite NLS through a flexible linker sequence $(\mathrm{G} / \mathrm{S} \times 10)^{12}$. These analyses revealed that neither the putative wild-type nor the mutated monopartite-like NLS fusion constructs were sufficient for nuclear import (Figure 3A-D). A detailed functional analysis of the canonical monopartite NLS motif identified that the second basic residue following the PXX (X, nonpolar residue) motif is necessary and required for nuclear import. ${ }^{13} \mathrm{In}$ ARID1A, this amino acid (position 1655) is not a basic residue, but is in fact glutamyl residue (PVLKQKK), which likely explains why this sequence is not a functional monopartite NLS. 
Motivated by these results, we sought to identify a functional NLS in ARID1A and utilized the NLS motif prediction tool, cNLS Mapper $^{9}$, restricting prediction constraints to motifs with a cNLS Mapper score of four or greater so as to include candidates that would confer cyto-nuclear as well as nuclear-only localization patterns. ${ }^{9}$ The output of this analysis identified a single, highconfidence bipartite NLS spanning residues 1368-1387 of ARID1A (Fig. 1) with a cNLS mapper score of 8 , indicating a high likelihood of nuclear targeting. To test whether this newly identified bipartite NLS is functional, we generated an ARID1A expression construct containing threonyl residues in place of the basic residues at positions 1369,1370 , and 1383 . These mutations were selected based on previous evidence indicating that both n-terminal and c-terminal basic amino acid motifs within the canonical bipartite NLS are required for nuclear import. ${ }^{13}$ This ARID1A expression construct harboring a mutant bipartite NLS was transiently expressed in ARID1A null cells (TOV21G and HEC1A) and ARID1A localization was assessed by immunofluorescence. These results revealed a predominant cyto-nuclear distribution for the ARID1A construct harboring the mutant bipartite-like NLS (Fig. 2C and 2F), in stark contrast to wild-type ARID1A (Fig. 2A and 2D). We confirmed these findings using GFP constructs fused to either the putative wild-type bipartite ARID1A NLS (KRPMDGTYGPPAKRHEGE) or a mutated variant (TTPMDGTYGPPAKTHEGE) (Figure 3E-H). These analyses revealed that the putative wildtype bipartite NLS fusion targeted GFP to cell nucleus, but that the mutated bipartite ARID1A NLS fusions resulted in a cyto-nuclear distribution of GFP in both cell lines tested (Fig. 3E-H). These results indicate that regions spanning 1370-1387 of ARID1A (KRPMDGTYGPPAKRHEGE) encode a functional NLS mediating nuclear import of ARID1A. This motif is highly homologous to the classical bipartite NLS motif $[(\mathrm{K} / \mathrm{R})(\mathrm{K} / \mathrm{R}) \mathrm{X} 10$ - 
12(K/R)3/5]. ${ }^{9}$ We performed comparative sequence analyses of this ARID1A NLS sequence across a diverse series of metazoans and found high conservation of this region (Fig. 4A). Furthermore, there exists high conservation of this NLS sequence in the ARID1A paralog, ARID1B, which differs by only two amino acid residues at positions 1362 and 1366 (Fig. 4B). NLS prediction analyses of the human ARID1B sequence revealed a cNLS score of 10 for a highly homologous motif, i.e. KRHMDGMYGPPAKRHEGE, suggesting that this motif is likely to functions as an NLS sequence for ARID1B.

We further tested the stability of wild-type and bipartite NLS-mutated ARID1A by immunoblot analyses following proteasomal inhibition with MG132 treatment in HEC1A cells. Our results showed that wild-type ARID1A (nuclear localized) is more actively degraded as compared to ARID1A harboring a mutated bipartite NLS (cyto-nuclear localized) (Supplemental Fig. 1). These results are consistent with previous evidence indicating that cytoplasmically localized ARID1A exhibits greater stability than nuclear localized ARID1A. ${ }^{8}$

\section{Discussion}

The present work has defined a novel bipartite NLS in ARID1A that mediates nuclear import of this important nuclear tumor suppressor protein. Our experiments provide confirmatory evidence of NLS functionality using GFP constructs fused with the ARID1A bipartite wild-type or mutant NLS sequences. We further show that cyto-nuclear localized, bipartite NLS mutant ARID1A exhibits greater protein stability than nuclear-localized, wildtype ARID1A. These latter findings provide evidence to support that the cytoplasmic fraction of ARID1A exhibits greater protein stability than nuclear ARID1A fractions, reflecting previous evidence to this effect. ${ }^{8}$ We further 
show that this novel ARID1A bipartite NLS motif is highly conserved across lower metazoans, as well as in the ARID1B paralog. In addition to identification and functional characterization of a novel NLS in ARID1A, our results are potentially clinically relevant as, although loss of function mutations frequently manifest as a result of the production of a truncated and unstable protein, loss of function can also result from mutation of key domains responsible for protein trafficking. Recent studies suggest that tumors with ARID1A loss of function mutations respond differentially to specific therapies. Understanding the spectrum of loss of function in ARID1A will enhance precision medicine strategies to treat these malignancies. ${ }^{14-16}$ Further, these insights directly bear on the diagnostic utility of nuclear ARID1A absence or presence estimates by immunohistochemistry analyses, suggesting a combined strategy that employs both IHC and sequencing of ARIDIA should be considered the gold standard for determining ARID1A loss of function. This information adds substantially to a rapidly expanding knowledgebase regarding this frequently mutated tumor suppressor gene and contributes key functional information that will be important in further development and understanding of the spectrum of mutations that manifest in loss of function of this frequently mutated nuclear tumor suppressor. 


\section{Acknowledgements}

Funding for this work was from the United States Medical Research Acquisition Activity (W81XWH-11-2-013) and the Office of the Assistant Secretary of Defense for Health Affairs through the Department of Defense Ovarian Cancer Research Program (W81XWH-15-1-0065). The opinions, conclusions or assertions contained herein are the private views of the authors and are not to be construed as official or as reflecting the views of the department of the Army, Navy, Air Force, the Department of Defense or the United States Government.

\section{Conflict of Interest Statement}

The authors do not have any conflicts of interest to report. 


\section{Figure Legends}

Figure 1. Diagram of the human ARID1A protein with functional motifs and putative nuclear localization sequences (NLS) denoted. NLS sequence candidates derived from cNLS mapper $^{9}$ or from literature sources $(\mathrm{N} / \mathrm{A}) .^{8} *$ - NLS sequences tested and $* *$ confirmed in this work.

Figure 2. The bipartite NLS 1368-NYKRPMDGTYGPPAKRHEGE-1387 is a functional nuclear localization sequence for ARID1A. Immunofluorescence analysis of ARID1A ectopically-expressed in representative models of ARID1A mutated tumor cell lines, i.e. TOV21G (A-C) and HEC1A (D-F). A \& D: Transfected with wildtype ARID1A. B \& E: Transfected with monopartite NLS mutated ARID1A. C \& F: Transfected with bipartite NLS mutated ARID1A. Green: ARID1A, orange: F-Actin (Phalloidin), blue: nuclei (Hoechst 33342). Images are representative of three biological replicate experiments.

Figure 3. Validation of a novel, bipartite NLS in ARID1A using GFP-NLS fusion constructs. Immunofluorescence analysis of TOV21G (A, C, E and G) and HEC1A (B, D, F and $\mathrm{H}$ ) cells ectopically-expressing GFP and wildtype or mutated versions of candidate monopartite (A-D) or bipartite (E-H) ARID1A NLS motifs. Green: GFP, orange: F-Actin (Phalloidin), blue: nuclei (Hoechst 33342). Images are representative of three biological replicate experiments.

Figure 4. The bipartite NLS sequence, 1368-NYKRPMDGTYGPPAKRHEGE-1387, is highly conserved amongst metazoans and highly homologous to the human ARID1A paralog, ARID1B. A: ARID1A protein sequences were obtained from Uniprot for all available metazoan species and the entry with the longest protein sequence lacking multiple null residues, i.e. $\mathrm{XX}$ sequences, for each species were aligned using Clustal Omega (EMBL-EBI). Bipartite NLS sequence alignment was generated using Jalview ${ }^{17}$ and the composite sequence logo was 
generated using weblogo ${ }^{18}$. B: Blast analysis of the human ARID1A bipartite NLS sequence, 1368-NYKRPMDGTYGPPAKRHEGE-1387, aligned to ARID1B (NP_059989.2). 


\section{References}

1. Nie, Z., Xue, Y., Yang, D., Zhou, S., Deroo, B. J., Archer, T. K., and Wang, W. (2000) A specificity and targeting subunit of a human SWI/SNF family-related chromatinremodeling complex, Mol Cell Biol 20, 8879-8888.

2. Chandler, R. L., Brennan, J., Schisler, J. C., Serber, D., Patterson, C., and Magnuson, T. (2013) ARID1a-DNA interactions are required for promoter occupancy by SWI/SNF, Mol Cell Biol 33, 265-280.

3. Van Rechem, C., Boulay, G., and Leprince, D. (2009) HIC1 interacts with a specific subunit of SWI/SNF complexes, ARID1A/BAF250A, Biochem Biophys Res Commun 385, 586590.

4. Huang, J., Zhao, Y. L., Li, Y., Fletcher, J. A., and Xiao, S. (2007) Genomic and functional evidence for an ARID1A tumor suppressor role, Genes, Chromosomes and Cancer 46, 745-750.

5. Wang, X., Nagl Jr, N. G., Flowers, S., Zweitzig, D., Dallas, P. B., and Moran, E. (2004) Expression of p270 (ARID1A), a component of human SWI/SNF complexes, in human tumors, International journal of cancer 112, 636-642.

6. Lowery, W. J., Schildkraut, J. M., Akushevich, L., Bentley, R., Marks, J. R., Huntsman, D., and Berchuck, A. (2012) Loss of ARID1A-associated protein expression is a frequent event in clear cell and endometrioid ovarian cancers, Int J Gynecol Cancer 22, 9-14.

7. Cerami, E., Gao, J., Dogrusoz, U., Gross, B. E., Sumer, S. O., Aksoy, B. A., Jacobsen, A., Byrne, C. J., Heuer, M. L., Larsson, E., Antipin, Y., Reva, B., Goldberg, A. P., Sander, C., and Schultz, N. (2012) The cBio cancer genomics portal: an open platform for exploring multidimensional cancer genomics data, Cancer Discov 2, 401-404.

8. Guan, B., Gao, M., Wu, C. H., Wang, T. L., and Shih Ie, M. (2012) Functional analysis of inframe indel ARID1A mutations reveals new regulatory mechanisms of its tumor suppressor functions, Neoplasia 14, 986-993.

9. Kosugi, S., Hasebe, M., Tomita, M., and Yanagawa, H. (2009) Systematic identification of cell cycle-dependent yeast nucleocytoplasmic shuttling proteins by prediction of composite motifs, Proc Natl Acad Sci U S A 106, 10171-10176.

10. Jones, S., Wang, T. L., Shih, I. M., Mao, T. L., Nakayama, K., Roden, R., Glas, R., Slamon, D., Diaz, L. A., and Vogelstein, B. (2010) Frequent mutations of chromatin remodeling gene ARID1A in ovarian clear cell carcinoma, Science 330, 228.

11. Guan, B., Wang, T. L., and Shih Ie, M. (2011) ARID1A, a factor that promotes formation of SWI/SNF-mediated chromatin remodeling, is a tumor suppressor in gynecologic cancers, Cancer Res 71, 6718-6727.

12. Snapp, E. (2005) Design and use of fluorescent fusion proteins in cell biology, Curr Protoc Cell Biol Chapter 21, Unit 2124.

13. Makkerh, J. P., Dingwall, C., and Laskey, R. A. (1996) Comparative mutagenesis of nuclear localization signals reveals the importance of neutral and acidic amino acids, Curr Biol 6, 1025-1027.

14. Bitler, B. G., Fatkhutdinov, N., and Zhang, R. (2015) Potential therapeutic targets in ARID1A-mutated cancers, Expert opinion on therapeutic targets, 1-4.

15. Bitler, B. G., Aird, K. M., Garipov, A., Li, H., Amatangelo, M., Kossenkov, A. V., Schultz, D. C., Liu, Q., Shih Ie, M., Conejo-Garcia, J. R., Speicher, D. W., and Zhang, R. (2015) 
Synthetic lethality by targeting EZH2 methyltransferase activity in ARID1A-mutated cancers, Nature medicine 21, 231-238.

16. Shen, J., Peng, Y., Wei, L., Zhang, W., Yang, L., Lan, L., Kapoor, P., Ju, Z., Mo, Q., Shih Ie, M., Uray, I. P., Wu, X., Brown, P. H., Shen, X., Mills, G. B., and Peng, G. (2015) ARID1A Deficiency Impairs the DNA Damage Checkpoint and Sensitizes Cells to PARP Inhibitors, Cancer discovery 5, 752-767.

17. Waterhouse, A. M., Procter, J. B., Martin, D. M., Clamp, M., and Barton, G. J. (2009) Jalview Version 2--a multiple sequence alignment editor and analysis workbench, Bioinformatics 25, 1189-1191.

18. Schneider, T. D., and Stephens, R. M. (1990) Sequence logos: a new way to display consensus sequences, Nucleic Acids Res 18, 6097-6100. 


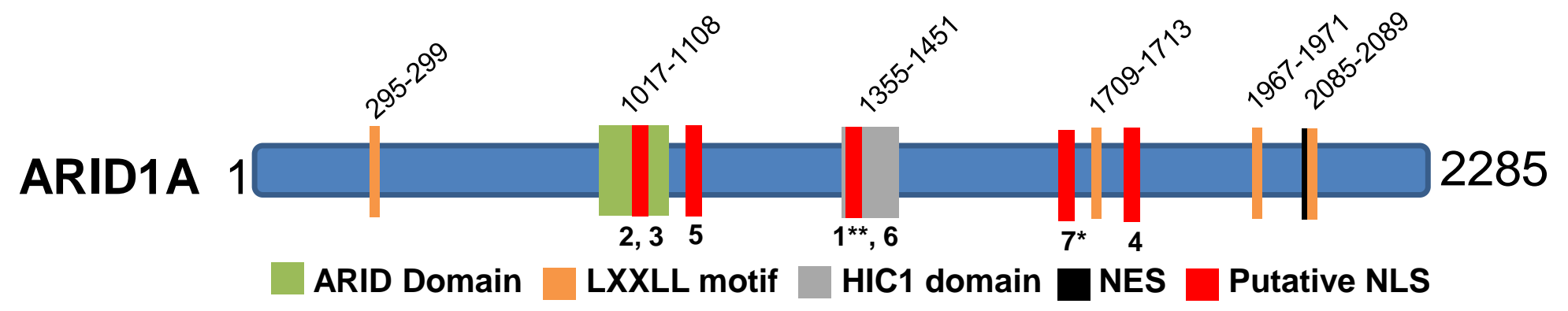

\begin{tabular}{|c|c|c|c|}
\hline $\begin{array}{c}\text { Candidate } \\
\text { Sequence }\end{array}$ & $\begin{array}{c}\text { Position } \\
\text { (aa) }\end{array}$ & $\begin{array}{c}\text { Putative Nuclear } \\
\text { Localization Sequence }\end{array}$ & $\begin{array}{c}\text { cNLS } \\
\text { Mapper } \\
\text { Score }\end{array}$ \\
\hline $1^{* *}$ & $1368-1387$ & NYKRPMDGTYGPPAKRHEGE & 8 \\
\hline 2 & $1046-1073$ & RKPLDLYRLYVSVKEIGGLTQVNKNKKW & 5.6 \\
\hline 3 & $1068-1097$ & NKNKKWRELATNLNVGTSSSAASSLKKQYI & 4.6 \\
\hline 4 & $1790-1818$ & FSGKDKPASENSEEKLISKFDKLPVKIVQ & 4.1 \\
\hline 5 & $1196-1228$ & DSTFQKRNSMTPNPGYQPSMNTSDMMGRMSYEP & 4 \\
\hline 6 & $1368-1396$ & NYKRPMDGTYGPPAKRHEGEMYSVPYSTG & N/A \\
\hline
\end{tabular}

*tested and **confirmed in this manuscript 


\section{Wild Type}

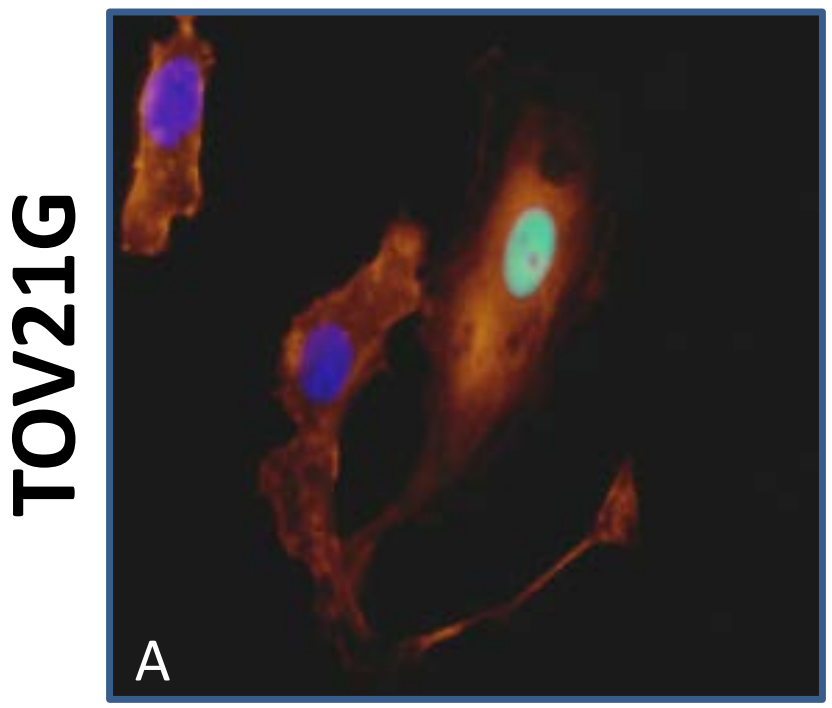

B

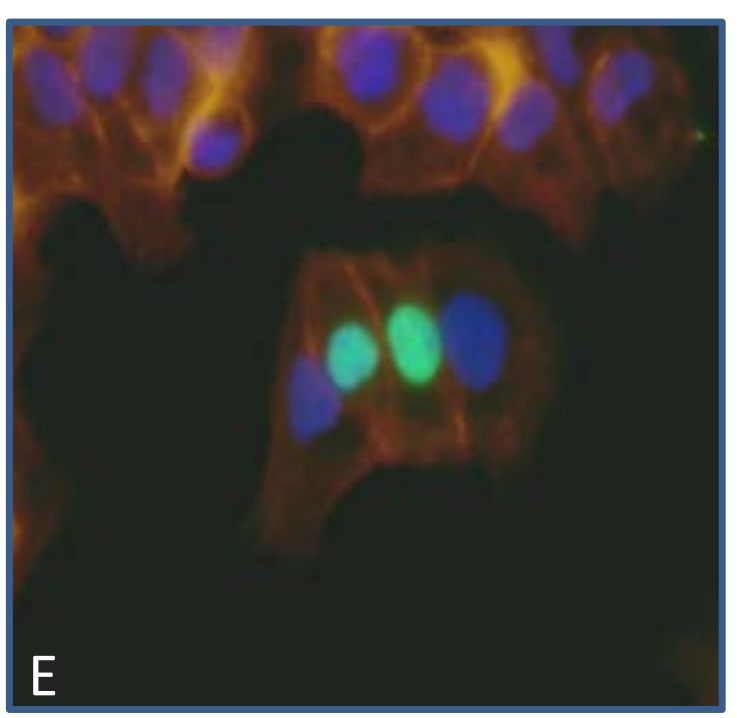

NLS \#1: PVLKQTTTLT

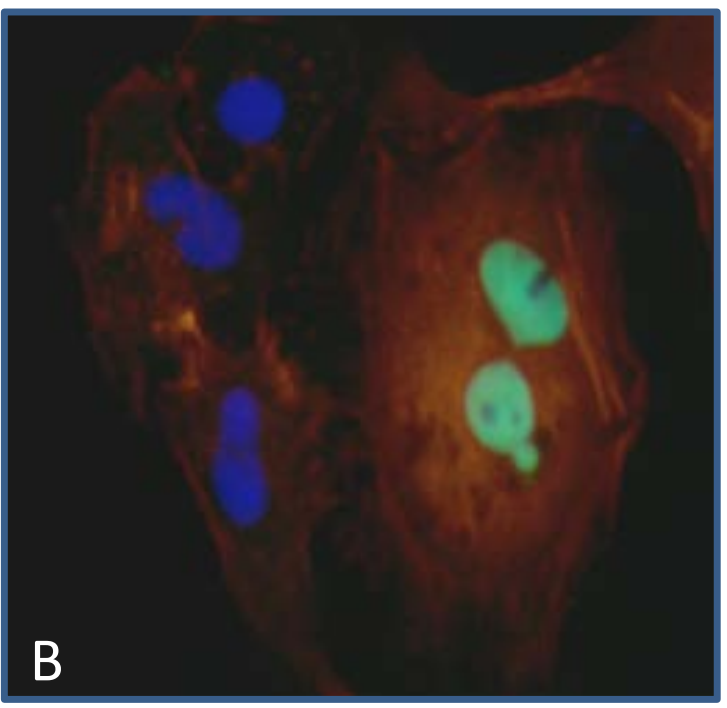

c

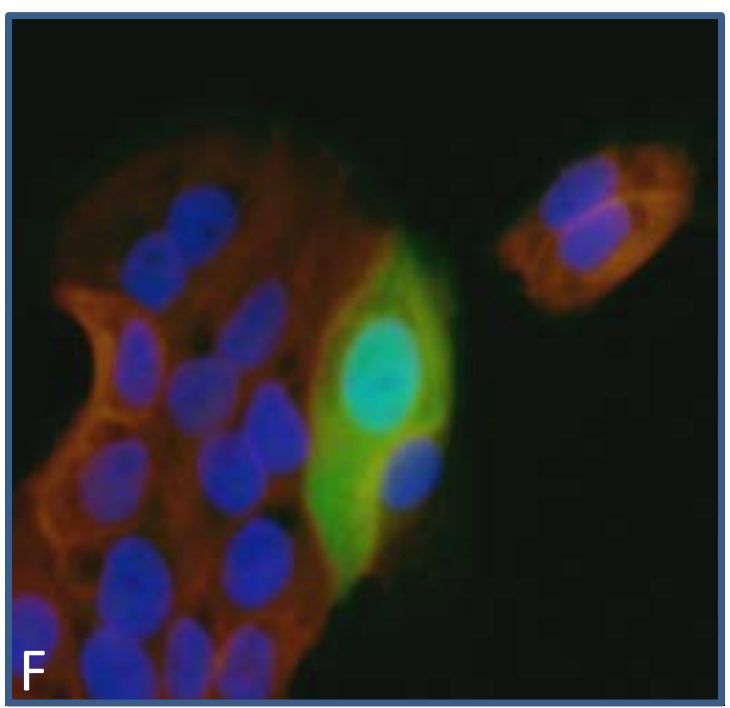

Bateman et al., Fig. 2 


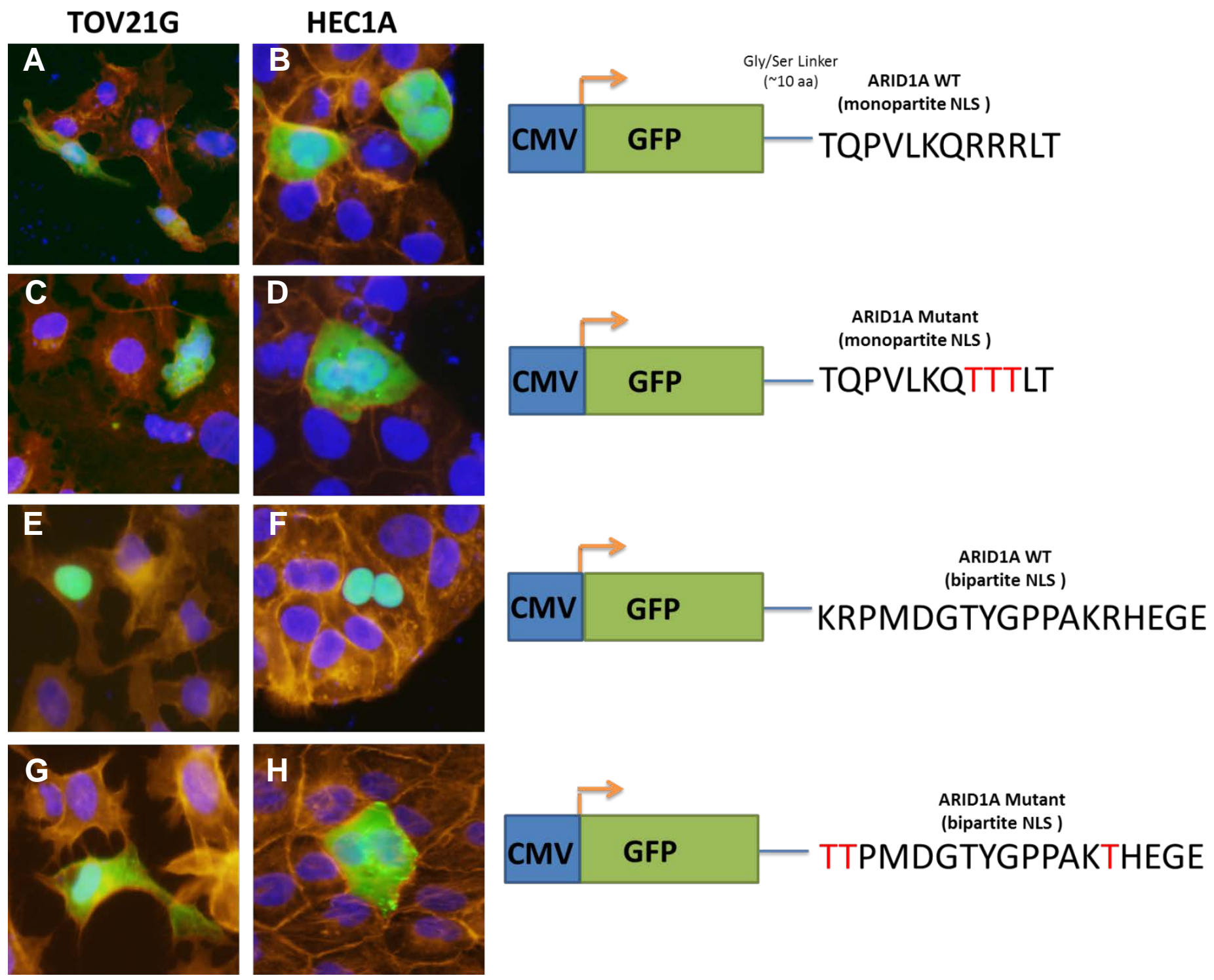

Bateman et al., Fig. 3 


\section{A. ${ }^{4-}$

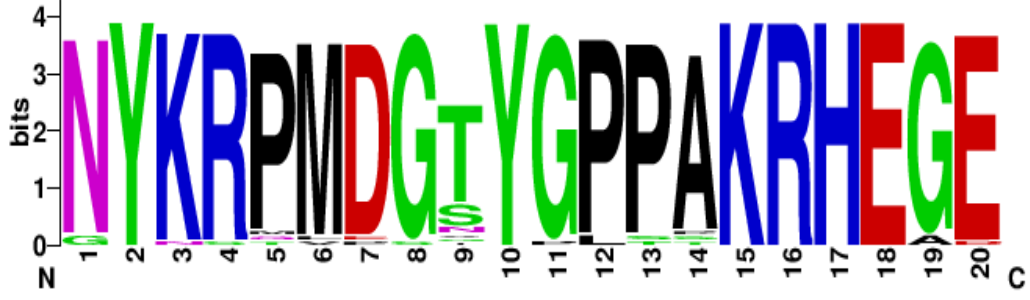

B.

ARID1A 1370KRPMDGTYGPPAKRHEG 1386

KR MDG YGPPAKRHEG

ARID1B 1360KRHMDGMYGPPAKRHEG 1376

YKRPMDGTYGPPAKRHEGE

tr|H2LHSO|H2LHSO ORYLA/1313-1330 GYKR P VE G GYPP P-SKRH EA tr|F7DC46|F7DC46 XENTR/1018-1037 N Y K R P MD G S Y G P S P K R H E G E tr|H3AK61|H3AK61_LATCH/984-1003 N Y K R QMD G S Y GP P T K R H E GE tr|R4G9F5|R4G9F5_ÁANOCA/1002-1021 N Y K R P MD G N Y GP P AKR H EAE trlV8PJ12|V8PJ12 OPHHA/945-964 N Y KR TMD G N Y GP P AKR R E GE tr|H9H1L5|H9H1L5 MELGA/998-1017 N Y K R PMD G S Y GP P AK R H E G E $t r|F 6 T 7 C 8| F 6 T 7 C 8$ M $O N D O / 1098-1117$ N Y KR P MD G T Y GP P AKR H E GE tr|G3WJS9|G3WJS9_SARHA1025-1044 N Y K R P MD G T Y G P P AK R H E GE $t r \mid$ G1RDV1|G1RDV1_NOMLE/1055-1074 N Y K R P MD G T Y GP P AK R H E G E tr|/3NOW3|/3NOW3 ICTTR/700-718 - YKR PMD G T Y GPPAKRH EGE tr|D4A3E3|D4A3E3 RAT/1106-1125 N Y KR P MD G T Y GP P AKR R E GE tr|G1SNP1|G1SNP1 RABIT/1080-1099 N Y K R P MD G T Y GP P AK R H E G E sp|A2BH4O|ARI1A_MOUSE/1369-1388 N Y K R P MD G T Y GP P AKR H E GE tr|U3FPA3|U3FPA3_CALJA1370-1389 N Y K R P MD G T Y GP P AK R H E G E Ir|G3QQK3|G3QQK3 GORGO/1266-1285 N Y K R P MD G T Y G P P AKR R E G E tr|H2N8F1|H2N8F1 PONAB/1117-1136 N Y K R P MD G T Y G P P AKR H E GE $t r \mid$ AOAOD9S841|AOAOD9S841_CHLSB/1319-1338 N Y K R P MD G T Y GP P AKR R E GE $t|| K 6 Z T Z 3 \mid K 6 Z T Z 3$ PANTR/1370-1389 N Y K R P MD G T Y GP P AK R H E G E tr|HOWKR8|HOWKR8_OTOGA/1006-1025 N Y K R P MD GAY GP P AKR R E GD trlHOVGS4|HOVGS4_CAVPO/989-1008 N Y K R P MD G T Y GP P AK R H E GE trlG1NYOO|G1NYOO-MYOLU/983-1002 N Y KR P MD S T Y GP TAKR H E GE

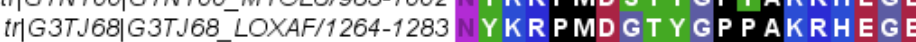
$t r|E 1 B 925| E 1 B 925 \_B O V I N / 1196-1215$ N Y K R P MD G T Y GP P A K R H E G E tr|W5P2D7|W5P2D7_SHEEP/988-1007 N Y K R P MD G T Y GP P AK R H E G E trIF7AAM8|F7AAM8 HORSE/988-1007 N Y K R P MD G T Y GP P AKR H E G E trlM3YW55|M3YW55 MUSPF/1120-1139 N Y K R P MD G T Y G P P AKR R E GE $t r|M 3 V K 12| M 3 V K 12$ PIG/1037-1056 N Y K R P MD G T Y GP P AK R H E G E tr|E2RSH9|E2RSH9_CANFA/1370-1389 N Y K R P MD G T Y GP P AK R H E G E tr|M3WHN6|M3WHN6 FELCA/989-1008 N Y K R PMD G T Y GP P AKR H E G E $t r|G 1 L W 62| G 1 L W 62$ All ME/989-1008 N Y K R P MD G T Y GP P AKR H E G E tr|U3/C42|U3/C42 F/CAL/954-973 N Y K R P MD G S Y G LP AKR R E GE tr|HOYRW3|HOYRW3 TAEGU/995-1014 N Y K R P MD G S Y GLP P A R H E G E tr|K7GHO9|K7GHO9_PELSI/994-1013 N Y K R P MD G T Y GP P AKR H E GE tr|U3/TJ4|U3/TJ4_ANAPL/985-1004 N Y K R PMD G S Y GP P AKR H E GE tIIT2MJQ4|T2MJQ4 HYDVU/734-742 G Y N SMLL P I -

tr|H9FGZ7|H9FGZ7_MACMU/167-166 